\title{
Equilibrated Residual Error Estimates are $p$-Robust
}

\author{
Dietrich Braess, Veronika Pillwein and Joachim Schöberl
}

November 24, 2007

\begin{abstract}
Equilibrated residual error estimators applied to high order finite elements are analyzed. The estimators provide always a true upper bound for the energy error. We prove that also the efficiency estimate is robust with respect to the polynomial degrees. The result is complete for tensor product elements. In the case of simplicial elements, the theorem is based on a conjecture, for which numerical evidence is provided.
\end{abstract}

Keywords: a posteriori error estimates, hypercircle method, $h p$ finite elements, equilibration

\section{Introduction}

Various a posteriori error estimates for numerical solutions with finite elements of low order are known to be reliable and efficient [13]. They are used to create locally refined meshes in an adaptive way that fit to the elliptic problem under consideration. Many theoretical and practical aspects have been considered in the literature.

The situation is less satisfactory for the $p$-version and the $h p$-version of the finite element method. Recently, Melenk and Wohlmuth [9] have shown for triangular and quadrilateral meshes that the efficiency of residual error estimators decrease only as $p^{-1}$ if the polynomial degree $p$ increases. They reported also, however, that numerical results do not admit to expect better theoretical results. 
In this paper we will turn to a posteriori error estimates for the $p$-method and the $h p$-method by the hypercircle method that may be traced back to Prager and Synge [10]. There is actual interest in this technique, since one obtains reliable bounds without generic constants in the dominant term and cheap implementations have been described recently; see, e.g., [4, 8]. Merely local problems have to be solved; they are organized on local patches around nodes of the mesh, while local problems for other classical error estimators are oriented to elements of the triangulation.

Numerical examples show that the new estimators do not suffer from a loss of efficiency for large $p$. There is a complete proof for rectangular and quadrilateral meshes while a conjecture for an ingredient has to be built in in the case of simplicial meshes. A crucial tool is a result on the right inverse of the divergence operator. It can be proven for rectangles and will be left as a conjecture for triangular partitions of the domain. The advantage of the estimator by the hypercircle method is certainly that it reflects the $H^{-1}$-norm of the residues while the well-known residual estimators refer to weighted $L_{2}$ norms of them. Of course, we have to pay for it in the analysis, and we have to deal with distributional forms of the differential operators.

The outline of the paper follows. Section 2 describes the postprocessing which yields the error estimate. Its reliability is an immediate consequence of Prager and Synge's theorem. Section 3 is concerned with the proof of the efficiency, which emphasizes the difference to residual error estimators. It contains a result on the right inverse of the divergence operator when distributional terms are included, and it may be of independent interest. A projector on univariate polynomials that is uniformly bounded in $p$ with respect to two norms is the topic of Section 4. The paper concludes with numerical examples.

\section{Formulation of the method}

Let $\Omega$ be a polygonal or polyhedral domain in $\mathbb{R}^{2}$ or $\mathbb{R}^{3}$. The variational formulation of an elliptic differential equation of second order in divergence form is written as

$$
a(u, v)=f(v) \quad \forall v \in V
$$

with

$$
a(u, v)=\int_{\Omega} a \nabla u \cdot \nabla v d x \quad f(v)=\int_{\Omega} f v d x .
$$


We consider the finite element approximation with polynomials of higher order on a quasi-uniform triangulation of $\Omega$ with simplicial, rectangular or hexahedral elements $T$. Since the notation for the finite element spaces differ for the triangulations above, we will focus on triangular meshes throughout the paper unless otherwise stated. The finite element solution is given by

$$
a\left(u_{N}, v\right)=f(v) \quad \forall v \in V_{N}
$$

and in this case we have

$$
V_{N}=\left\{v \in V: v_{\mid T} \in P^{p+1}(T)\right\} .
$$

We assume that $f$ is a piece-wise polynomial of degree $p$. Otherwise, $f$ is assumed to be the piecewise approximation of the exact $f_{e x}$. As usual, this additional approximation is left to the user, and the effect of the data oscillation $f-f_{e x}$ is assumed to produce an extra error of higher order, in particular, if $\int_{T}\left(f-f_{e x}\right) d x=0$ for each element $T$.

We assume that $a$ is piece-wise constant and for simplicity $a \approx 1$. The analysis could be extended to a highly varying coefficient $a$ satisfying the quasi-monotonicity condition.

We will establish a postprocessing algorithm which provides an equilibrated flux $\sigma \in \Sigma_{N} \subset H$ (div), i.e., the flux satisfies pointwise

$$
-\operatorname{div} \sigma=f .
$$

The difference between the discrete flux $a \nabla u_{N}$ and the postprocessed one provides a true upper bound without generic constant to the error measured in energy error. Specifically, by the theorem of Prager and Synge,

$$
\left\|u-u_{N}\right\|_{a} \leq\left\|a \nabla u_{N}-\sigma\right\|_{a^{-1}, L_{2}} ;
$$

cf. [10] or [3, Theorem III.5.1]. In the literature, also the names hypercircle method or two-energies principle are found.

Computations have shown that the overestimation of the error is bounded uniformly in the mesh-size as well as the polynomial degree. This means that the resulting estimator is efficient, and we will prove it in this paper.

The hypercircle method is the general framework. We propose the following specific construction for the flux $\sigma$ : Following [4] the residual $r \in V^{*}$ defined as

$$
\langle r, v\rangle:=f(v)-a\left(u_{N}, v\right)
$$


is written as

$$
\langle r, v\rangle=\sum_{T}\left(r^{T}, v\right)_{0, T}+\sum_{E}\left(r^{E}, v\right)_{0, E} .
$$

The well known element and edge residuals are

$$
\begin{aligned}
r^{T} & =f+\operatorname{div}\left(a \nabla u_{N}\right), \\
r^{E} & =\left[a \partial_{n} u_{N}\right] .
\end{aligned}
$$

Here the divergence is taken pointwise on each element. Let $\phi_{i}$ denote the hat basis function associated with the vertex $x_{i}$. Its support is $\omega_{i}=\cup\{T$ : $\left.x_{i} \in \partial T\right\}$. We decompose the residual by this partition of unity, i.e.,

$$
\left\langle r_{\omega_{i}}, v\right\rangle:=\left\langle r, \phi_{i} v\right\rangle
$$

Recalling (??) we get the element and edge terms of the local residual:

$$
\begin{aligned}
& r_{\omega_{i}}^{T}=\phi_{i}\left\{f+\operatorname{div}\left(a \nabla u_{N}\right)\right\} \\
& r_{\omega_{i}}^{E}=\phi_{i}\left[a \partial_{n} u_{N}\right] .
\end{aligned}
$$

The Galerkin orthogonality of the hat basis functions yields

$$
\left\langle r_{\omega_{i}}, 1\right\rangle=\left\langle r, \phi_{i}\right\rangle=0
$$

i.e., the local residuals are bi-orthogonal to constant functions.

The element terms as well as edge terms in (6) are polynomials of degree at most $p+1$. We construct a vector function $\sigma_{\omega_{i}}$ in the broken RaviartThomas space $R T^{p+1}\left(\omega_{i}\right)[1]$,

$$
\begin{aligned}
R T_{-1}^{p}\left(\omega_{i}\right) & :=\left\{\tau \in L_{2}\left(\omega_{i}\right):\left.\tau\right|_{T} \in R T^{p}(T), T \subset \omega_{i}\right\} \\
\text { with } \quad R T^{p}(T) & :=\left\{\tau: \tau(x)=q_{T}+s_{T} x, q_{T} \in\left(P^{p}\right)^{2}, s_{T} \in P^{p}\right\}
\end{aligned}
$$

such that

$$
\operatorname{div} \sigma_{\omega_{i}}=r_{\omega_{i}}
$$

The divergence is understood in distributional sense and is consistent with (4). Combining this with the bounding condition, it translates into

$$
\begin{array}{rlrl}
\operatorname{div}_{T} \sigma_{\omega_{i}} & =r_{\omega_{i}}^{T} & & \text { in } T \subset \omega_{i}, \\
{\left[\sigma_{\omega_{i}} \cdot n\right]} & =r_{\omega_{i}}^{E} & \text { at } E \subset \omega_{i}, \\
\sigma_{\omega_{i}} \cdot n=0 & & \text { on } \partial \omega_{i} .
\end{array}
$$


The vector function $\sigma_{\omega_{i}}$ with minimal $L_{2}$-norm corresponds to the gradient that minimizes the complementary energy and is given by the solution of a mixed method. Specifically, we can use the implementation of the RaviartThomas element by Arnold and Brezzi [1]. The implementation refers to the broken Raviart-Thomas space; we have only to insert the inhomogeneous condition $(8)_{2}$ instead of the homogeneous one: $\left[\sigma_{\omega_{i}} \cdot n\right]=0$. The stability is independent of the right hand side of the equations and has been proven in [1]. We refer to [5] for elements of Raviart-Thomas type or BDM elements on quadrilateral grids or for three-dimensional domains.

The computation of these local fluxes is the crucial step of the equilibration. By adding up all the fluxes we obtain the global correction

$$
\sigma^{\Delta}=\sum \sigma_{\omega_{i}}
$$

as a solution of

$$
\operatorname{div} \sigma^{\Delta}=r
$$

The postprocessed flux is $\sigma:=a \nabla u_{N}+\sigma^{\Delta}$, and from (2) it follows that

$$
\left\|\sigma^{\Delta}\right\|_{a^{-1}}^{2}
$$

is an error estimate without a generic constant.

The main result of the present paper is to prove the $p$-robust efficiency of the error estimate.

Theorem 1 ( $p$-robust efficiency). If the mesh consists of

- affine quadrilateral or hexahedral elements

- or triangular or tetrahedral elements and Conjecture 6 is valid

then the error estimator is locally efficient, i.e.,

$$
\left\|\sigma_{\omega_{i}}\right\| \leq c\left\|\nabla u-\nabla u_{N}\right\|_{a, \omega_{i}}
$$

holds with a constant $c$ that is independent of $h$ and $p$.

Remark 2. A global correction with the same divergence as $\operatorname{div} \sigma^{\Delta}$ can be constructed by fluxes in $R T^{p}$ instead of $R T^{p+1}$. Since $\sum_{i} \phi_{i}=1$, the sums 
$\sum_{i} r_{\omega_{i}}^{T}$ and $\sum_{i} r_{\omega_{i}}^{E}$ are polynomials of maximal degree $p$. Therefore we can replace the local residuals by the orthogonal projections onto $P^{p}$ and setting

$$
\begin{aligned}
r_{\omega_{i}}^{T} & =\Pi^{p}\left(\phi_{i}\left\{f+\operatorname{div}\left(a \nabla u_{N}\right)\right\}\right), \\
r_{\omega_{i}}^{E} & =\Pi^{p}\left(\phi_{i}\left[a \partial_{n} u_{N}\right]\right) .
\end{aligned}
$$

Numerical experiments show that the efficiency is reduced only slightly in this way. Similarly, the use of the BDM elements slightly improves the efficiency due to the larger number of local degrees of freedom.

\section{Proof of the efficiency}

First we note that the $H^{-1}\left(\omega_{i}\right)$-norm of the local residual is bounded by the local error:

$$
\begin{aligned}
\left\|r_{\omega_{i}}\right\|_{H^{-1}\left(\omega_{i}\right)} & =\sup _{v \in H_{0}^{1}\left(\omega_{i}\right),\|v\|_{1} \leq 1}\left\langle r_{\omega_{i}}, v\right\rangle \\
& =\sup _{v}\left\langle r, \phi_{i} v\right\rangle=\sup _{v} a\left(u-u_{N}, \phi_{i} v\right) \\
& \leq\left\|u-u_{N}\right\|_{a} \sup _{v \in H_{0}^{1}} \frac{\left\|\phi_{i} v\right\|_{a}}{\|\nabla v\|_{L_{2}\left(\omega_{i}\right)}} \\
& \leq c\left\|u-u_{N}\right\|_{a} .
\end{aligned}
$$

The last inequality follows from

$$
\left\|\phi_{i} v\right\|_{a} \leq c\left\|\nabla\left(\phi_{i} v\right)\right\|_{0} \leq\|\nabla v\|_{0}^{2}+\left\|\nabla \phi_{i}\right\|_{0}^{2}\|v\|_{0}^{2} \leq c\|v\|_{H^{1}}
$$

for $v \in H_{0}^{1}\left(\omega_{i}\right)$. Here we used an inverse inequality for $\nabla \phi_{i}$ and the properly scaled Friedrichs inequality on the element patch.

We will get at our aim, i.e., the efficiency as stated in inequality (9), when we show the existence of a $\sigma_{\omega_{i}}$ on the patch such that

$$
\operatorname{div} \sigma_{\omega_{i}}=r_{\omega_{i}} \quad \text { and } \quad\left\|\sigma_{\omega_{i}}\right\|_{0, \omega_{i}} \leq C\left\|r_{\omega_{i}}\right\|_{H^{-1}} .
$$

This means that we have to find a continuous right inverse of the divergence that applies to distributions of the form (4) on the patch.

The constructive proof will differ from the construction given in the previous section for the use in actual computations. The estimates are based on two ingredients: One is the right-inverse of the divergence on one element, the other one is the extension of normal-traces from edges to elements that has been treated in [7] and is given in the following lemma. 
Lemma 3 (Polynomial extension operators).

Let $\gamma \subset \partial T$ be the union of one, two, or three edges of $T$. Let $g_{n} \in L_{2}(\gamma)$ be given such that $\left.g_{n}\right|_{E} \in P^{p}(E)$. If $\gamma=\partial T$ we additionally assume $\int_{\gamma} g_{n}=0$. Then there exists an extension $\sigma_{p} \in R T^{p}(T)$ such that

$$
\operatorname{div} \sigma_{p}=0 \quad \text { and } \quad \operatorname{tr}_{n, \gamma} \sigma_{p}=g_{n},
$$

and

$$
\left\|\sigma_{p}\right\|_{0} \preceq \inf _{\substack{\sigma \in L_{2}(T) \\ \operatorname{div} \sigma=0, \operatorname{tr} n, \gamma=g_{n}}}\|\sigma\|_{0} .
$$

A major contribution of this paper is to construct a right inverse of the divergence on quadrilateral and hexahedral elements. An essential tool is a projection operator onto univariate polynomials that is uniformly bounded in $p$ for two norms.

Lemma 4. Let $I=(-1,1)$. There exist projection operators $Q^{p}: L_{2}(I) \rightarrow$ $P^{p}$ which are uniformly bounded in $p$ with respect to $L_{2}$ and simultaneously the $H^{1}$-norm.

The proof of the lemma is postponed to the next section.

We turn to rectangular grids. Here $P^{k, \ell}$ contains the polynomials of degree $k$ in the first variable and degree $\ell$ in the second one. The Raviart-Thomas elements on rectangular grids build the spaces $R T^{[k]}:=P^{k+1, k} \times P^{k, k+1}$.

Theorem 5 (right inverse on tensor product elements).

Let $T$ be a square or a cube. Let $r_{T} \in P^{p, p}(T)$. Then there exists a $\sigma_{T} \in$ $R T^{[p]}(T)$ such that

$$
\operatorname{div} \sigma_{T}=r_{T} \quad \text { and } \quad\left\|\sigma_{T}\right\|_{0, T} \leq c\left\|r_{T}\right\|_{H^{-1}(T)} .
$$

Proof. We restrict ourselves to the 2D-case and consider the Dirichlet problem

$$
\begin{aligned}
\Delta w=r_{T} & \text { in } T, \\
w=0 & \text { on } \partial T .
\end{aligned}
$$

The flux $\sigma:=\nabla w$ satisfies $\operatorname{div} \sigma=r_{T}$ and $\|\sigma\|_{0}=\left\|r_{T}\right\|_{-1}$. We have to project $\sigma$ into the polynomials. Take the projector $Q=Q^{p+1}$ from Lemma 4 and define another projector onto $P^{p}$ by

$$
\widetilde{Q} v:=(Q I v)^{\prime}
$$




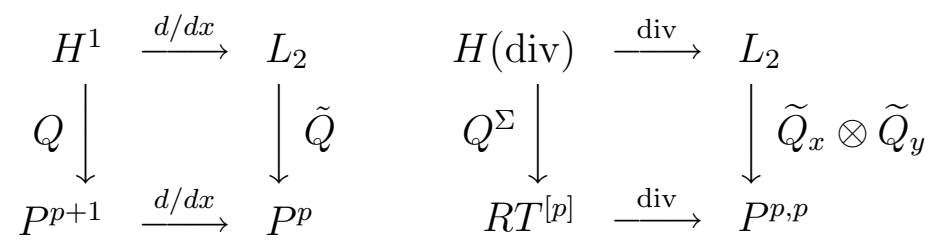

Commuting diagram properties of projectors

with $I v(x):=\int_{-1}^{x} v(s) d s$. The relation

$$
\|\widetilde{Q} v\|_{0}=\left\|(Q I v)^{\prime}\right\|_{0} \leq\left\|(I v)^{\prime}\right\|_{0}=\|v\|_{0}
$$

shows that $\widetilde{Q}$ is bounded in the $L_{2}$-norm. The two operators have the commuting diagram property

$$
\widetilde{Q} u^{\prime}=(Q u)^{\prime} .
$$

The tensor product projector

$$
Q^{\Sigma}=\left(Q_{x} \otimes \widetilde{Q}_{y}\right) \times\left(\widetilde{Q}_{x} \otimes Q_{y}\right): L_{2}(T) \rightarrow R T^{[p]}(T)
$$

is bounded in $L_{2}(T)$, and it has the commuting diagram property with the divergence, i.e.,

$$
\operatorname{div} Q^{\Sigma}=\left(\widetilde{Q}_{x} \otimes \widetilde{Q}_{y}\right) \operatorname{div} .
$$

We set $\sigma_{T}=Q^{\Sigma} \sigma$ to complete the proof of the lemma.

The corresponding result for BDM elements is obvious.

At the moment, an analogous result for the right inverse on simplicial elements can be posed only as a conjecture. Numerical computations with finite elements of high order and, of course, the result for rectangles support this conjecture.

Conjecture 6 (right inverse on simplicial elements).

Let $T$ be a triangular or tetrahedral element. Let $r_{T} \in P^{p}(T)$. Then there exists a $\sigma_{T} \in R T^{p}(T)$ such that

$$
\operatorname{div} \sigma_{T}=r_{T} \quad \text { and } \quad\left\|\sigma_{T}\right\|_{0, T} \preceq\left\|r_{T}\right\|_{\left[H_{0}^{1}(T)\right]^{*}} .
$$

For a further support of the conjecture we have computed the constants $C_{p, q}$ in Table 1 such that the inequalities

$$
\min _{\substack{\sigma_{T} \in B D M^{p+1} \\ \operatorname{div} \sigma_{T}=r_{T}}}\|\sigma\|_{0}^{2} \leq C_{p, q} \sup _{\substack{v \in P^{p+q} \cap H_{0}^{1}(T)}} \frac{\left(v, r_{T}\right)^{2}}{\|v\|_{H^{1}}^{2}}
$$


hold for all $r_{T} \in P^{p}$. The constants can be computed by finding the largest eigenvalue of generalized eigenvalue problems. The discrete $H^{-1}$-norms in (10) approaches the $H^{-1}$-norm from below. Hence,

$$
\min _{\substack{\sigma_{T} \in B D M p+1 \\ \operatorname{div} \sigma_{T}=r_{T}}}\|\sigma\|_{0}^{2} \leq C_{p}\left\|r_{T}\right\|_{\left[H_{0}^{1}(T)\right]^{*}}^{2}
$$

with $C_{p} \leq C_{p, q}$. The results indicate that $C_{p}$ is bounded in $p$.

\begin{tabular}{c|ccc} 
Table 1: Coefficients $C_{p q}$ in $(10)$ \\
\hline $\mathrm{p}$ & $\mathrm{q}=3$ & $\mathrm{q}=5$ & $\mathrm{q}=8$ \\
\hline 1 & 1.81 & 1.76 & 1.76 \\
2 & 2.05 & 1.92 & 1.92 \\
4 & 2.43 & 1.99 & 1.99 \\
8 & 3.23 & 2.00 & 2.00 \\
16 & 4.92 & 2.38 & 2.00 \\
\hline
\end{tabular}

Now we turn to the main theorem of the paper that guarantees the efficiency of the a posteriori error estimate for large polynomial degrees and may be also of independent interest. Here we will focus on triangular grids although we have to base the analysis now on the conjecture above.

Theorem 7. Let $\omega$ be the patch of elements around the vertex $V$. Let $r$ be the residual

$$
\langle r, v\rangle=\sum_{T \subset \omega} \int_{T} r_{T} v+\sum_{E \subset \omega} \int_{E} r_{E} v
$$

with $r_{T} \in P^{p}(T)$ and $r_{E} \in P^{p}(E)$. Moreover assume that $\langle r, 1\rangle=0$. Then there holds

$$
\inf _{\substack{\sigma \in R T_{-1,0}^{p} \\ \operatorname{div} \sigma=r}}\|\sigma\|_{0} \leq C\|r\|_{\left[H^{1}(\omega)\right]^{*}}
$$

and the constant $C$ is independent of $p$.

Proof. Step 1. Elimination of element residuals.

For each element $T \subset \omega$ we construct $\sigma_{T} \in R T^{p}(T)$ such that $\operatorname{div}_{T} \sigma_{T}=$ $-r_{T}$. By Theorem 5 this is possible with

$$
\left\|\sigma_{T}\right\|_{0, T} \preceq\left\|r_{T}\right\|_{H_{0}^{1}(T)^{*}} .
$$


Now we estimate $\left\|r_{T}\right\|_{H_{0}^{1}(T)^{*}}$, and the non-locality of negative norms will be no problem. Given $v \in H_{0}^{1}(T)$ with $\|v\|_{1, T} \leq 1$, there is an extension by zero to $\tilde{v} \in H^{1}(\omega)$ and $\left(r_{T}, v\right)_{0, T}=\langle r, \tilde{v}\rangle \leq\|r\|_{H^{1}(\omega)^{*}}\|\tilde{v}\|_{1, \omega}$. Hence, $\left\|r_{T}\right\|_{H_{0}^{1}(T)^{*}} \leq\|r\|_{H^{1}(\omega)^{*}}$ and

$$
\left\|\sigma_{T}\right\|_{0, \omega} \preceq\|r\|_{H^{1}(\omega)^{*}}
$$

Let $\sigma^{(1)}:=\sum \sigma_{T}$ and

$$
r^{(1)}:=r-\operatorname{div} \sum_{T} \sigma_{T},
$$

with the divergence operator understood in the distributional sense. The new residual $r^{(1)}$ contains only edge terms including the edges on $\partial \omega$. Since we have $\|\operatorname{div} s\|_{H^{1}(\omega)^{*}} \leq\|s\|_{0, \omega}$ for all $s \in L_{2}(\omega)^{2}$, it follows from (13) that the modified functional is bounded

$$
\left\|r^{(1)}\right\|_{H^{1}(\omega)^{*}} \preceq\|r\|_{H^{1}(\omega)^{*}} .
$$

Moreover, recalling that (14) is understood in the distributional sense, we have

$$
\left\langle\operatorname{div} \sigma_{T}, 1\right\rangle=-\int_{\partial T} \sigma_{T} \cdot n 1+\int_{T} \operatorname{div}_{T} \sigma_{T} 1=-\int_{T} \sigma \nabla 1=0
$$

and again $\left\langle r^{(1)}, 1\right\rangle=0$.

Step 2. Elimination of boundary edge residuals.

For each triangle $T$ consider the boundary-value problem

$$
\begin{aligned}
-\Delta w & =0, & & \\
w & =0 & & \text { on internal edges, } \\
\partial_{n} w & =r_{E}^{(1)} & & \text { for edges on } \partial T \cap \partial \omega,
\end{aligned}
$$

or in weak form with $w \in V_{T}:=\left\{v \in H^{1}(T): v=0\right.$ on internal edges $\}$,

$$
(\nabla w, \nabla v)=\int_{E \subset \partial \omega} r_{E}^{(1)} v
$$


Set $\sigma_{T}=\nabla w$ in this step (without changing the symbol). From (15) it follows that

$$
\begin{aligned}
\left\|\sigma_{T}\right\|_{0, T} & =\|\nabla w\|_{0, T}=\sup _{v \in V_{T}} \frac{\int_{E \subset \partial \omega} r_{E}^{(1)} v}{\|\nabla v\|}=\sup _{v \in \tilde{V}_{T}} \frac{\left\langle r^{(1)}, v\right\rangle}{\|\nabla v\|} \\
& \leq\left\|r^{(1)}\right\|_{H^{1}(\omega)^{*}},
\end{aligned}
$$

where $\tilde{V}_{T}$ contains the extension of functions in $V_{T}$ onto $\omega$ by zero.

Since $r_{E}^{(1)}$ is a polynomial, by Lemma 3 there exists a polynomial $\sigma_{T}^{(2)}$ such that

$$
\operatorname{tr}_{n, E} \sigma_{T}^{(2)}=r_{E}^{(1)}
$$

on $E \subset \partial \omega$, and $\left\|\sigma_{T}^{(2)}\right\|_{0, T} \preceq\left\|\sigma_{T}\right\|_{0, T} \preceq\|r\|_{H^{1}(\omega)^{*}}$. This construction can be done independently triangle by triangle. Next we subtract the divergences of $\sigma^{(2)}:=\sum_{T} \sigma_{T}^{(2)}$ to obtain

$$
r^{(2)}=r^{(1)}-\operatorname{div} \sum_{T} \sigma_{T}^{(2)}
$$

By construction, $r^{(2)}$ contains only edge residuals on internal edges. Moreover, $\left\langle r^{(2)}, 1\right\rangle=0$, and

$$
\left\|r^{(2)}\right\|_{H^{1}(\omega)^{*}} \preceq\|r\|_{H^{1}(\omega)^{*}}
$$

Step 3. Elimination of internal edge residuals.

We will circle around the patch: $E_{1}, T_{1}, E_{2}, T_{2}, \ldots, E_{n}, T_{n}, E_{1}$; cf. Figure 1 . We start with triangle $T_{1}$. Choose

$$
V_{T}=\left\{v \in H^{1}(T): v=0 \text { on } E_{2}\right\} .
$$

We pose the problem for $w \in V_{T}$

$$
(\nabla w, \nabla v)=\int_{E_{1}} r_{E_{1}}^{(2)} v
$$

A function $v \in V_{T}$ can be extended to $\tilde{v} \in H^{1}(\omega)$ with $\operatorname{supp} \tilde{v} \subset T_{1} \cup T_{n}$ and $\|\tilde{v}\|_{1, \omega} \leq c\|v\|_{1, T}$, where $c$ depends only on the shape parameter. By construction,

$$
\int_{E_{1}} r_{E_{1}} v=\left\langle r^{(2)}, \tilde{v}\right\rangle
$$




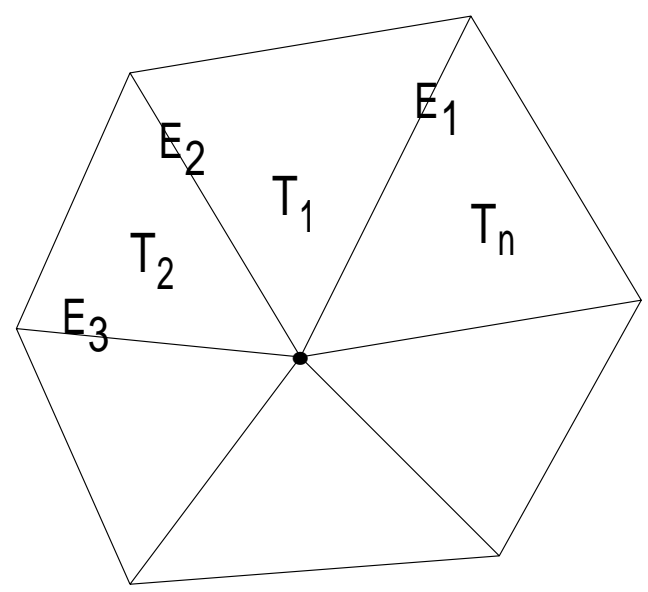

Figure 1. Enumeration of triangles and edges in the patch $\omega$ with $n$ triangles.

We set $\sigma_{T}=\nabla w$, and conclude as above

$$
\begin{aligned}
\left\|\sigma_{T}\right\|_{0} & \preceq\left\|r^{(2)}\right\|_{H^{1}(\omega)^{*}}, \\
\operatorname{div} \sigma_{T} & =0, \\
\operatorname{tr}_{n, \partial \omega} \sigma_{T} & =0 \quad \text { on } \partial T_{1} \cap \partial \omega \\
\operatorname{tr}_{n, E_{1}} \sigma_{T} & =r_{E, 1} .
\end{aligned}
$$

By Lemma 3 there exists a polynomial $\sigma_{T_{1}}^{3}$ with the same properties, and bounded as $\sigma_{T}$. By subtracting, we obtain the new residual

$$
r^{(3,1)}=r^{(2)}-\operatorname{div} \sigma_{T_{1}}^{3} .
$$

It vanishes also on edge $E_{1}$.

We can now proceed with this procedure for all triangles in the patch except the last one. On the last triangle the residual is reduced to the edge terms on the two adjacent edges in $\omega$. Here, we pose a pure Neumann problem. It is solvable, since $\left\langle r^{(3, n-1)}, 1\right\rangle=0$.

We have decomposed the residual as a sum of divergences of piecewise polynomials that are bounded as stated in the theorem. 


\section{A one dimensional projection operator}

In order to prove Lemma 4 we start with Legendre polynomials $P_{i}$ which are $L_{2}$-orthogonal on $(-1,+1)$ :

$$
\left(P_{i}, P_{j}\right):=\left(P_{i}, P_{j}\right)_{L_{2}(-1,1)}=\delta_{i, j} \frac{2}{2 i+1} .
$$

We define the projection operator

$$
\left(T_{i} u\right)(x)=\frac{2 i+1}{2} \int_{-1}^{1} P_{i}(y) u(y) d y P_{i}(x),
$$

which sends a function $u \in L^{2}(-1,1)$ to the $i$ th term of its Legendre expansion. Hence,

$$
\left\|T_{i} u\right\|_{0}^{2}=\left(\frac{2 i+1}{2}\right)^{2}\left(P_{i}, u\right)^{2} \int_{-1}^{1} P_{i}(x)^{2} d x=\frac{2 i+1}{2}\left(P_{i}, u\right)^{2}
$$

and, for $u \in L_{2}(-1,1)$

$$
\sum_{i=0}^{\infty}\left\|T_{i} u\right\|_{0}^{2}=\|u\|_{0}^{2}
$$

The following quasi-projection operator into the space of polynomials of degree $2 p-1$ was introduced by de la Vallée-Poussin [6]:

$$
S_{p}=\sum_{i=0}^{2 p-1} c_{i} T_{i} \quad \text { with } \quad c_{i}= \begin{cases}1, & i \leq p \\ (2 p-i) / p, & p<i \leq 2 p-1\end{cases}
$$

Lemma 8. The smoothing operators $S_{p}$ satisfy

(i) $S_{p}$ reproduce polynomials up to the order $p$.

(ii) The operators $S_{p}$ are uniformly bounded in $p$, i.e.,

$$
\left\|S_{p}\right\|_{L_{2}}=1 \quad \text { and } \quad\left\|S_{p}\right\|_{H^{1}} \leq 3
$$

Proof. The first assertion follows from the fact that $\left(T_{i} u\right)(x)$ gives the $i$ th term in the Legendre expansion of $u(x)$. So, if $u$ is a polynomial of degree less or equal $p$, then $\left(T_{i} u\right)(x)=0$ for all $i>p$ and we have $\left(S_{p} u\right)(x)=u(x)$. 
Next we estimate the $L^{2}$-norm of $S_{p}$. Note that $c_{i} \leq 1$. Exploiting the $L^{2}$-orthogonality of Legendre polynomials and identity (16) we calculate

$$
\begin{aligned}
\left\|S_{p} u\right\|_{0}^{2} & =\sum_{i, j=0}^{2 p-1} c_{i} c_{j} \frac{2 i+1}{2} \frac{2 j+1}{2}\left|\left(P_{i}, u\right) \|\left(P_{j}, u\right)\right|\left(P_{i}, P_{j}\right) \\
& =\sum_{i=0}^{2 p-1} c_{i}^{2}\left\|T_{i} u\right\|_{0}^{2} \leq \sum_{i=0}^{2 p-1}\left\|T_{i} u\right\|_{0}^{2}=\|u\|_{0}^{2} .
\end{aligned}
$$

In the course of estimating the $H^{1}$ seminorm of $S_{p} u$ we will use a wellknown relation between Legendre polynomials and their derivatives [12]

$$
P_{n}(x)=\frac{1}{2 n+1}\left(P_{n+1}^{\prime}(x)-P_{n-1}^{\prime}(x)\right)
$$

and perform partial integration

$$
\int_{-1}^{1} P_{i}(y) u(y) d y=-\frac{1}{2 i+1} \int_{-1}^{1}\left(P_{i+1}(y)-P_{i-1}(y)\right) u^{\prime}(y) d y .
$$

The boundary terms above vanish since $P_{n}(1)=1$ and $P_{n}(-1)=(-1)^{n}$. Another identity we will need is

$$
\left(P_{i}^{\prime}, P_{j}^{\prime}\right)= \begin{cases}0, & i-j \equiv_{2} 1, \\ l(l+1), & i-j \equiv_{2} 0 \text { where } l=\min \{i, j\} .\end{cases}
$$

Now we are in the position to start calculating

$$
\begin{aligned}
\left\|S_{p} u\right\|_{1}^{2} & =\sum_{i, j=1}^{2 p-1} c_{i} c_{j} \frac{(2 i+1)(2 j+1)}{4}\left(P_{i}^{\prime}, P_{j}^{\prime}\right)\left(P_{i}, u\right)\left(P_{j}, u\right) \\
& =\frac{1}{4} \sum_{i, j=1}^{2 p-1} c_{i} c_{j}\left(P_{i}^{\prime}, P_{j}^{\prime}\right)\left(P_{i+1}-P_{i-1}, u^{\prime}\right)\left(P_{j+1}-P_{j-1}, u^{\prime}\right) \\
& =: \frac{1}{4} \sum_{i, j=0}^{2 p} M_{i, j}\left(P_{i}, u^{\prime}\right)\left(P_{j}, u^{\prime}\right) .
\end{aligned}
$$

With this reordering performed in the last step we defined a symmetric matrix 
$M$ and we continue estimating,

$$
\begin{aligned}
4\left\|S_{p} u\right\|_{1}^{2} & \leq \sum_{i, j=0}^{2 p}\left|M_{i, j} \|\left(P_{i}, u^{\prime}\right)\right|\left|\left(P_{j}, u^{\prime}\right)\right| \\
& =\sum_{i, j=0}^{2 p} \frac{2\left|M_{i, j}\right|}{\sqrt{2 i+1} \sqrt{2 j+1}}\left\|T_{i} u^{\prime}\right\|_{0}\left\|T_{j} u^{\prime}\right\|_{0} .
\end{aligned}
$$

Let $M^{(0)}$ be the normalized matrix with entries $M_{i, j}^{(0)}=\frac{1}{\sqrt{2 i+1} \sqrt{2 j+1}}\left|M_{i, j}\right|$ and let $t=\left(\left\|T_{i} u^{\prime}\right\|_{0}\right)_{i=0}^{2 p}$. Then we have

$$
2\left\|S_{p} u\right\|_{1}^{2} \leq t^{T} M^{(0)} t \leq \rho\left(M^{(0)}\right)\|t\|_{2}^{2}=\rho\left(M^{(0)}\right) \sum_{i=0}^{2 p}\left\|T_{i} u^{\prime}\right\|_{0}^{2} \leq \rho\left(M^{(0)}\right)\left\|u^{\prime}\right\|_{0}^{2}
$$

Thus it remains to show that the spectral radius of $M^{(0)}$ is bounded by a constant. Therefore we will estimate the row-sum norm of $M^{(0)}$ which is an upper bound for $\rho\left(M^{(0)}\right)$.

We omit the lengthy computations of the coefficients of $M$ and will merely state the resulting expressions. Furthermore, because of identity (19) only the coefficients $M_{i, j}$ with $i \equiv_{2} j$ are nonzero.

We list the results for $M$ starting with the diagonal entries,

$$
\begin{aligned}
M_{i, i} & =2(2 i+1), \quad 0 \leq i \leq p-1, \\
M_{p, p} & =\frac{1}{p^{2}}(p-1)\left(4 p^{2}-p-2\right), \\
M_{i, i} & =\frac{1}{p^{2}}\left[(2 p-i-1)^{2}(4 i+2)+4 i(i-1)\right], \\
M_{2 p, 2 p} & =\frac{2}{p}(2 p-1 \leq i \leq 2 p-1,
\end{aligned}
$$

Since $M$ is symmetric, we will only consider the upper right triangular matrix. So from now on we assume $i \leq j-2$. First, we have in the upper left block

$$
M_{i, j}=0, \quad \text { for } \quad i<j \leq p-1 .
$$

Since also $M_{i, j}=0$ if $i-j$ is odd, we silently assume in the following formulas that $i-j$ is even. In order to deal with a special factor in the last row, we 
introduce the factor

$$
\theta_{j}= \begin{cases}1, & j \leq 2 p-1, \\ \frac{1}{2}, & j=2 p .\end{cases}
$$

The nonzero off-diagonal entries are now given by

$$
\begin{aligned}
M_{i, p} & =-\frac{2}{p}(2 i+1), \quad i<p, \\
M_{i, j} & =-\frac{4}{p}(2 i+1) \theta_{j}, \quad i<p<j, \\
M_{p, j} & =-\frac{2}{p^{2}}(p-1)(3 p+2) \theta_{j}, \quad p<j, \\
M_{i, j} & =\frac{4}{p^{2}}\left(1+2 i+3 i^{2}-2 p(2 i+1)\right) \theta_{j}, \quad p<i<j .
\end{aligned}
$$

Now we can verify by inspection that

$$
M_{i, i} \leq 2(2 i+1) \quad \text { and } \quad\left|M_{i, j}\right| \leq \frac{4}{p} \sqrt{2 i+1} \sqrt{2 j+1}, i \neq j .
$$

Since there are at most $p$ nonzero off-diagonal entries in each row, it follows that the rowsum of $M^{(0)}$ does not exceed 6, and the proof is complete.

It remains to find a projector from $P^{2 p-1}$ to $P^{p}$. This will be done by separating polynomials with zero boundary values. To this end we define extension operators that provide polynomials of low $H^{s}$ norm, $s=0,1$, to given boundary data:

$$
E_{p}^{(s)} u(x)=\underset{\substack{v \in P^{p}(-1,1), v(-1)=u(-1), v(1)=u(1)}}{\operatorname{argmin}}\|v\|_{s}, \quad s=0,1 .
$$

The operators have the following properties:

Lemma 9. There is a constant $C$ independent of $p$ such that

$$
\left\|E_{p}^{(s)} u\right\|_{s} \leq C\|u\|_{s}, \quad \forall u \in P^{2 p-1}, s=0,1 .
$$

Proof. We restrict ourselves to $p \geq 2$, since we may choose $E_{p}^{(s)} u=u$ if $p=0$ or $p=1$.

For $s=1$ the minimal energy extension is given by

$$
E_{p}^{(1)} u(x)=u(-1) \frac{1-x}{2}+u(1) \frac{1+x}{2} .
$$


The norm estimate follows by the trace theorem, so we have

$$
\left\|E_{p}^{(1)} u\right\|_{1} \leq|u(-1)|+|u(1)| \leq C\|u\|_{1} .
$$

In order to determine the $L_{2}$-extension we consider the minimization for left and right endpoint separately,

$$
w^{ \pm}=\underset{v \in P^{p}(-1,1), v( \pm 1)=1, v(\mp 1)=0}{\operatorname{argmin}}\|v\|_{0}^{2} .
$$

It will be crucial that the estimate in [11, Lemma 9.1] is sharp. The ansatz $v(x)=\sum_{i=0}^{p} v_{ \pm, i} P_{i}(x)$ transforms the constraint minimization problems into algebraic ones,

$$
\min v^{T} A v \text {, }
$$

with the diagonal matrix $A=\operatorname{diag}\left(\frac{2}{2 i+1}\right)_{i=0}^{p}$. The constraints are now $\sum_{i=0}^{p}(-1)^{i} v_{-, i}=1$ and $\sum_{i=0}^{p} v_{-, i}=0$ for the left endpoint, respectively $\sum_{i=0}^{p} v_{+, i}=1$ and $\sum_{i=0}^{p}(-1)^{i} v_{+, i}=0$ for the right endpoint. Obviously the solutions are

$$
v_{+, i}=\frac{2 i+1}{p(p+2)}\left[1+\frac{(-1)^{i+p+1}}{p+1}\right] \quad \text { and } \quad v_{-, i}=(-1)^{i} v_{+, i} .
$$

The total extension operator is then given by

$$
E_{p}^{(0)} u(x)=w^{-}(x)+w^{+}(x)=\sum_{i=0}^{p}\left[(-1)^{i} u(-1)+u(1)\right] v_{+, i} P_{i}(x) .
$$

Exploiting the $L_{2}$-orthogonality of Legendre polynomials a simple summation shows that

$$
\begin{aligned}
\left\|E_{p}^{(0)} u\right\|_{0}^{2}= & \frac{2}{(p+1)(p+2)}\left[u(-1)^{2}+u(1)^{2}\right] \\
& +\frac{2}{p(p+1)(p+2)}\left[u(1)-(-1)^{p} u(-1)\right]^{2} .
\end{aligned}
$$

Examining (20) we find that $\left\|E_{p}^{(0)} u\right\|_{0} \leq 3\left\|E_{2 p-1}^{(0)} u\right\|_{0}$. The $L_{2}$-norm of the given function is certainly not smaller than the minimal one in $P^{2 p-1}$ and thus we obtain

$$
\left\|E_{p}^{(0)} u\right\|_{0} \leq 3\left\|E_{2 p-1}^{(0)} u\right\|_{0} \leq 3\|u\|_{0} .
$$


We note that it is necessary to restrict the domain of the extension operators in Lemma 8, e.g., to $P^{2 p-1}$. Otherwise we have no bound like (21). For this reason the map $S_{p}$ will enter into the analysis.

Now we define projection operators for functions with zero boundary values. Let

$$
L_{i}(x)=c_{i} \int_{-1}^{x} P_{i-1}(s) d s, \quad i \geq 2,
$$

where $c_{i}=\frac{1}{2} \sqrt{(2 i-3)(2 i-1)(2 i+1)}$, be the $i$ th integrated Legendre polynomial. These polynomials are orthogonal with respect to the $H^{1}$ norm and have zeros at $x= \pm 1$. The normalization has been chosen according to [2], where the the following norm estimates for $u=\sum_{i=2}^{M} u_{i} L_{i}$ have been shown:

$$
\left\|u^{\prime}\right\|_{0}^{2} \approx \sum_{i=2}^{M} i^{2} u_{i}^{2}, \quad\|u\|_{0}^{2} \approx \sum_{i=2}^{M}\left[\frac{1}{i^{2}} u_{i}^{2}+\left(u_{i}-u_{i+2}\right)^{2}\right] .
$$

We set $P_{0}^{k}=P^{k} \cap H_{0}^{1}$ and define the projection operators

$$
\begin{array}{rll}
R_{p} & : & P_{0}^{2 p-1} \rightarrow P_{0}^{p} \\
u=\sum_{i=2}^{2 p-1} u_{i} L_{i} & \mapsto \quad R_{p} u=\sum_{i=2}^{p}\left(u_{i}-\frac{i}{p} u_{2 p-i+1}\right) L_{i} .
\end{array}
$$

Lemma 10. The norms $\left\|R_{p}\right\|_{0}$ and $\left\|R_{p}\right\|_{1}$ of the projection operators are uniformely bounded in $p$.

Proof. Recalling (22) we obtain by a straight forward calculation

$$
\begin{aligned}
\left\|R_{p} u\right\|_{1}^{2} & \approx \sum_{i=2}^{p} i^{2}\left(u_{i}-\frac{i}{p} u_{2 p-i+1}\right)^{2} \\
& \leq 2\left(\sum_{i=2}^{p} i^{2} u_{i}^{2}+\sum_{i=2}^{p} \frac{i^{4}}{p^{2}} u_{2 p-i+1}^{2}\right) \\
& \leq 2\left(\sum_{i=2}^{p} i^{2} u_{i}^{2}+\sum_{i=2}^{p}(2 p-i+1)^{2} u_{2 p-i+1}^{2}\right) \\
& =2 \sum_{i=2}^{2 p-1} i^{2} u_{i}^{2} \leq C\|u\|_{1}^{2} .
\end{aligned}
$$


The boundedness in the $L^{2}$-norm follows the same lines. We first use (22) to express $\left\|R_{p} u\right\|_{0}$, and then basic estimates yield

$$
\begin{aligned}
\left\|R_{p} u\right\|_{0}^{2} \preceq & \sum_{i=2}^{p} \frac{1}{i^{2}} u_{i}^{2}+\sum_{i=2}^{p} \frac{1}{p^{2}} u_{2 p-i+1}^{2} \\
& +\sum_{i=2}^{p}\left(u_{i}-u_{i+2}\right)^{2}+\sum_{i=2}^{p} \frac{i^{2}}{p^{2}}\left(u_{2 p-i+1}-u_{2 p-i+3}\right)^{2} \\
\preceq & \sum_{i=2}^{2 p-1} \frac{1}{i^{2}} u_{i}^{2}+\left(u_{i}+u_{i+2}\right)^{2} \approx\|u\|_{0}^{2} .
\end{aligned}
$$

Now we are in the position to define the composite projection operators

$$
\tilde{R}_{p}^{(s)}=R_{p}\left(I-E_{p}^{(s)}\right)+E_{p}^{(s)}, \quad s=0,1 .
$$

Since $\left(E_{p}^{(1)}-E_{p}^{(0)}\right) v$ is a polynomial of degree less or equal $p$ for $v \in P^{2 p-1}$, the operators $\tilde{R}_{p}^{(0)}$ and $\tilde{R}_{p}^{(1)}$ coincide. Indeed,

$$
\begin{aligned}
\tilde{R}_{p}^{(1)} v & =\left[R_{p}\left(I-E_{p}^{(1)}\right)+E_{p}^{(1)}\right] v \\
& =\left[R_{p}\left(I-E_{p}^{(0)}\right)+E_{p}^{(0)}\right] v+\left(I-R_{p}\right)\left(E_{p}^{(1)}-E_{p}^{(0)}\right) v \\
& =\tilde{R}_{p}^{(0)} v .
\end{aligned}
$$

Hence, the norm estimates of the individual operators prove the estimate for $\tilde{R}_{p}^{(0)}=\tilde{R}_{p}^{(1)}$.

Finally we set $Q^{p}:=\tilde{R}_{p}^{(0)} S_{p}=\tilde{R}_{p}^{(1)} S_{p}$ to complete the proof of Lemma 4.

\section{Numerical example}

The Poisson equation $-\Delta u=1$ is considered on the L-shaped domain $\Omega:=(-1,+1)^{2} \backslash[0,1) \times(-1,0]$. We assume homogeneous Neumann boundary conditions on $\Gamma_{N}:=(0,1) \times\{0\}$ and homogeneous Dirichlet boundary conditions on $\Gamma_{D}:=\partial \Omega \backslash \Gamma_{N}$.

We have computed finite element solutions for $p=1,2,4$, and 8 with $h$-adaptive codes. Moreover, a reference solution was obtained by computations with even higher order polynomials. The equilibrated fluxes for the 
a posteriori error estimates have been determined with $\mathrm{BDM}^{p}$ and $\mathrm{BDM}^{p+1}$ elements. There are only small differences between the two variants. The efficiency was measured by the quotients

$$
\frac{\text { eror estimator }}{\text { real eror }} \text {. }
$$

They were smaller than 1.6 in all cases depicted in Figures 2 and 3. A selection is listed in Tables 2 and 3.

Table 2: Error of finite elements of degree 4 via $\mathrm{BDM}^{5}$.

\begin{tabular}{r|lll}
\hline unknowns & estimator & real error & quotient \\
\hline 45 & 1.165 & 0.8502 & 1.371 \\
97 & 1.011 & 0.7576 & 1.334 \\
191 & 0.9728 & 0.7491 & 1.299 \\
779 & 0.5313 & 0.4056 & 1.310 \\
1563 & 0.2139 & 0.1621 & 1.329 \\
3131 & 0.03393 & 0.02576 & 1.317 \\
4307 & 0.009050 & 0.007139 & 1.268 \\
\hline
\end{tabular}

Table 3: Error of finite elements of degree 8 via BDM ${ }^{9}$.

\begin{tabular}{r|lll}
\hline unknowns & estimator & real error & quotient \\
\hline 153 & 0.7873 & 0.5684 & 1.385 \\
353 & 0.6779 & 0.5047 & 1.343 \\
1009 & 0.5618 & 0.4253 & 1.321 \\
2561 & 0.3579 & 0.2695 & 1.328 \\
4113 & 0.2265 & 0.1701 & 1,331 \\
6441 & 0.1135 & 0.08516 & 1.332 \\
8769 & 0.05677 & 0.04259 & 1.333 \\
10321 & 0.03577 & 0.02683 & 1.333 \\
12649 & 0.01789 & 0.01342 & 1.333 \\
\hline
\end{tabular}




\section{References}

[1] D.N. Arnold and F. Brezzi, Mixed and nonconforming finite element methods: implementation, postprocessing and error estimates. $\mathrm{M}^{2} \mathrm{AN}$ 19 (1985) 7-32.

[2] S. Beuchler, A domain decomposition preconditioner for $p$-FEM discretizations of two-dimensional elliptic problems. Computing, 74 (2005) 299-317.

[3] D. Braess, Finite Elements: Theory, Fast Solvers and Applications in Solid Mechanics. Cambridge University Press, 3rd edition, 2007.

[4] D. Braess and J. Schöberl, Equilibrated residual error estimator for Maxwell's equations. Math. Comp. (to appear).

[5] F. Brezzi and M. Fortin, Mixed and Hybrid Finite Element Methods. Springer-Verlag, Berlin - Heidelberg - New York 1991.

[6] Ch. J. de la Vallée-Poussin, Leçons sur l'approximation des fonctions d'une variable réelle. Paris: Gauthier-Villars 1919.

[7] L. Demkowicz, J. Gopalakrishnan, and J. Schöberl, Polynomial extension operators (in preparation).

[8] R. Luce and B. Wohlmuth, A local a posteriori error estimator based on equilibrated fluxes. SIAM J. Numer. Anal. 42 (2004) 1394-1414.

[9] J.M. Melenk and B.I. Wohlmuth, On residual-based a posteriori error estimation in hp-FEM. Adv. Comput. Math. 15 (2001) 311-331.

[10] W. Prager and J.L. Synge, Approximations in elasticity based on the concept of function spaces. Quart. Appl. Math. 5 (1947) 241-269.

[11] C. Schwab and M. Suri, The optimal p-version approximation of singularities on polyhedra in the boundary element method. SIAM J. Numer. Anal. 33 (1996) 729-759.

[12] G. Szegö, Orthogonal Polynomials. AMS Colloquium Publications, Volume XXIII. 3rd edition, 1974. 


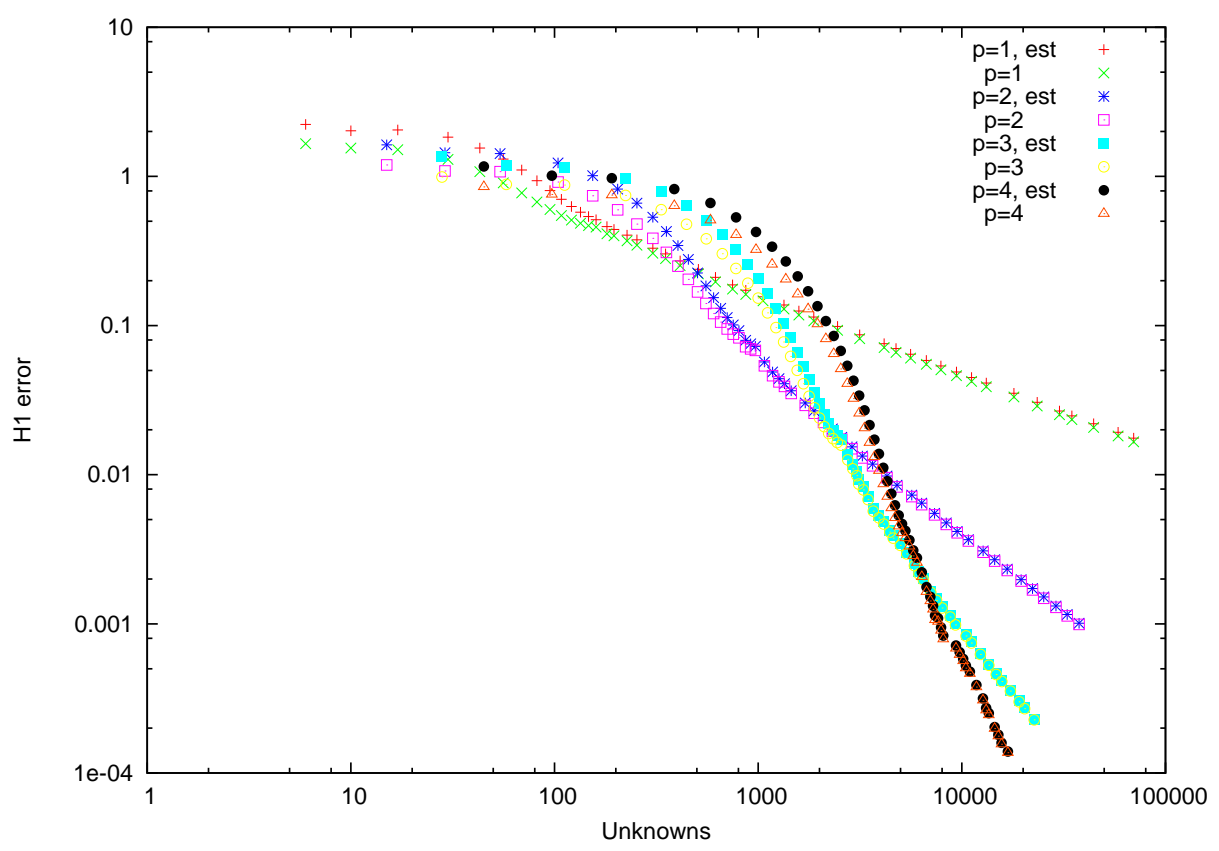

Figure 2. Results for L-shaped domain. The equilibration is performed with $\mathrm{BDM}^{p+1}$ elements. are

[13] R. Verfürth, A Review of A Posteriori Error Esimation and Adaptive Mesh-Refinement Techniques. Wiley-Teubner, Chichester - New York Stuttgart 1996.

Faculty of Mathematics, Ruhr-University, D 44780 Bochum, Germany email: Dietrich.Braess@ruhr-uni-bochum.de

Research Institute for Symbolic Computation, Johannes Kepler University, Altenbergerstrasse 69, A-4040 Linz, Austria email: Veronika.Pillwein@sfb013.uni-linz.ac.at

Center for Computational Engineering Science, RWTH Aachen University, D 52062 Aachen, Germany

email: Joachim.Schoeberl@mathcces.rwth-aachen.de 


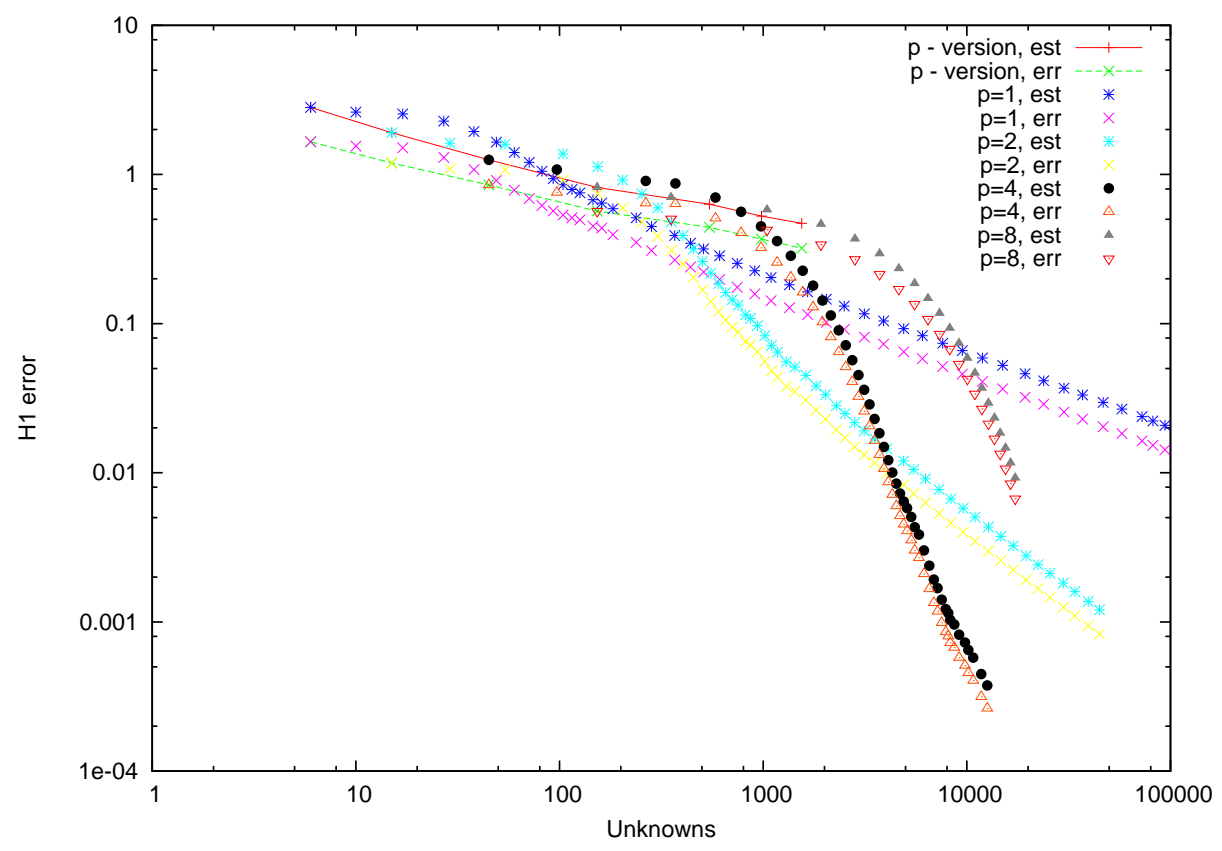

Figure 3. Results for L-shaped domain. The equilibration is performed with $\mathrm{BDM}^{p}$ elements. 